\title{
ANALYTICAL MODELING FOR NANOSTRUCTURE QUANTUM WELLS WITH EQUISPACED ENERGY LEVELS IN SEMICONDUCTOR TERNARY ALLOYS $\left(A_{x} B_{1-x} C\right)$
}

\author{
A. I. Ejere ${ }^{1, *}$, S. Okunzuwa ${ }^{2}$ and R. E. Oloko ${ }^{3}$ \\ 1, 2, DePARTMENT OF PHYSICS, UNIVERSITY OF BENIN, BENIN CITY, EDO STATE, NIGERIA. \\ 3, DEPARTMENT OF PHYSICS, UNIVERSITY OF PORT HARCOURT, CHOBA, RIVERS STATE, NIGERIA. \\ E-mail addresses; ${ }^{1}$ ejere.arthur@uniben.edu, ${ }^{2}$ okunzuwas@yahoo.com, ${ }^{3}$ rolioloko@yahoo.com
}

\begin{abstract}
The purpose of this study is to formulate an Analytical model of equispaced energy levels quantum wells (QWs) in semiconductor ternary alloys $\left(A_{x} B_{1-x} C\right)$. The procedure is by mapping the envelop function Schrodinger equation for realistic $Q W$, with the local conduction band edge as the potential experienced by an electron in the $Q W$ into an effective mass Schrodinger equation with a linear harmonic oscillator potential by the method of coordinate transformation. The electron effective mass and potential are then obtained as the signature for the equispaced energy level for QWs in semiconductor ternary alloys.
\end{abstract}

Keywords: Semiconductor nanostructures, Ternary alloys, Quantum wells, Equispaced energy levels, Effective mass.

\section{INTRODUCTION}

Confinement of electrons in nanostructures gives rise to quantum effects [1-3]. One of these effects is that experimental measurement involving these confined electrons are influenced by their environment and consequently prone to errors $[4,5]$ such as decoherence [6-8]. Consequently, many of the application of these nanostructures especially those that require emission and absoption are often affected by decoherence which usually affect quantum system. The nano-structuring of semiconductor materials was first introduced by Shockley [9] and later by Kroemer [10], however the progress of nanofabrication technology was affected by decoherence [9-12]. This has instigated the search for designs which may reduced the decoherence which cannot in principle be eliminated for the obvious reasons of the size of the nanostructure and that there is no physically isolated quantum system in nature $[6,13]$. One possible way to reduced the effect of decoherence may be to design semiconductors nanostructure with equispaced energy levels which will lead to coherent emission and absorption.
The mapping of the Schrodinger equation set up for the semiconductor nanostructure into appropriate dimensional simple harmonic oscillator (SHO) by coordinate transformation approach yield equispaced energy levels in a QW and the eigenfunction are given by the well-known hermite polynomias. Since the goal of physics is to account for all physical phenomena, irrespective of their complexities, with the few known ones, mapping of semiconductors nanostructure into the SHO will enable us to obtain it's appropriate solution for the effective mass function $\mathrm{m}(\mathrm{z})$, potential function $v(z)$, grading function $x(z)$ and the electron wave function $u(z)$ are signature for the equispaced energy levels for the semiconductor ternary alloys QWs [14-17] the reasons for calculating the effective masses is because they can be used as inputs for further single band calculations so as to obtain the electronic structure when the absolute size of the structures is known [23] further, the carrier effective masses of a QW is strongly connected to the carrier mobility, is one of the most important devices parameters. The reason being that low carrier effective mass means the parent materials is highly 
suitable for high speed carrier mobility applications. Our choice of ternary alloys QWs for this preliminary study is motivated not only by the need to generalized previous studies $[16,17]$ of ID for the semiconductor ternary alloys ( $\left.A_{x} B_{1-x} C\right)$ but also because many of the physical effect in quantum well structures can be seen at room temperature and can be exploited in real device.

\section{ANALYTICAL MODELLING}

For an electron confined in a QW, the Schrodinger equation is given as,

$$
\begin{aligned}
& \frac{-\eta^{2}}{2 m} \frac{d^{2} \psi}{d z^{2}}+V(z)=E \psi \\
& E_{n}=\frac{-\eta^{2}}{2 m}\left[\frac{n \pi}{L_{z}}\right]^{-2} \text { and } \psi_{n}=A \sin \left[\frac{n \pi z}{L_{z}}\right]
\end{aligned}
$$

where $n=1,2,3, \ldots$

In Eq. (1), $V(z)$ is the structural potential (i.e. the "quantum well" potential) seen by the electron in the well with confinement in the $z$ direction, $m$ is the electron effective mass, and $E$ and $\psi$ are the eigenenergy and eigenfunction respectively.

Eq. (2) is an ideal case solution of treating the QW (with $L_{z}$ as well width) as a well with infinite barrier potential well. This solution is not realistic because of the increase effect of decoherence.

In the semiconductor ternary alloy $A_{x} B_{1-x} C$, the $x$ is the mole fraction of the semiconductor alloy composition. It is the grading function which linearly depends on the conduction band edge hence the potential $V(z)$ experienced by the electron in the QW;

$$
x(z)=\frac{V(z)}{\Delta V}
$$

Where $\Delta \mathrm{V}$, is the potential difference between the materials $A B$ and $B C$.

The $x(z)$ is also related to the electron effective mass $\mathrm{m}(\mathrm{z})$ as

$$
V(z)=\phi\left[m(z)-m_{B C}\right] / \Delta m
$$

where $\Delta m=m_{B C}$ is the difference of effective mass between the materials $A B$ and $B C$.

The $x(z)$ is also related to the electron effective mass $m(z)$ as:

$$
V(z)=\phi\left[m(z)-m_{B C}\right]
$$

Further, to conserve the electron flux, we have to choose barrier continuous case $\frac{1}{m} \frac{d \psi}{d z}$ which will lead to envelop function Schrodinger equation for realistic QW with a position dependent effective mass related to the position dependent potential $[14,18]$.

$$
\frac{-\eta^{2}}{2 m} \frac{d}{d z^{2}}\left(\frac{1}{m_{z}} \frac{d \psi}{d z}\right)+\phi\left[m(z)-m_{B C}\right] \psi=E \psi
$$

We seek the function $m(z), x(z)$ and $V(z)$ such that the energy spectrum of (6) has equidistant states as one dimensional SHO $[14,19]$. For inconvenience the following unit are used energy in eV length in A and effective mass in free electron mass. Taking into accounts these units Eq.(6) becomes

$\frac{d}{d z}\left(\frac{1}{m_{z}} \frac{d \psi}{d z}\right)+q\{E-\phi\}\left[m(z)-m_{B C}\right] \psi=0$.

Now if we introduce the coordinate transformation $z=g(y)$ and consequently introduce a new function $u(y)[20,21]:$

$u(y)=\psi(y) \exp \left[-\frac{1}{2} \int_{y o}^{y} \frac{1}{m g^{\prime}} \frac{d m g^{\prime}}{d y} d y\right]$

Eq. (7) becomes

$\frac{d^{2} u}{d y^{2}}+\left[A(y)+q m g^{\prime 2}\left\{E-\phi\left[m(z)-m_{B C}\right]\right\}\right]=0$

where

$A(y)=\frac{1}{2} \frac{d}{d y}\left[\frac{1}{m g^{\prime}} \frac{d m g^{\prime}}{d y}\right]-\frac{1}{4}\left[\frac{1}{m g^{\prime}} \frac{d m g^{\prime}}{d y}\right]^{2}$

The potential for the one Dimension (ID) SHO denoting equispaced energy level is given by $[14,15]$.

$$
V=\frac{1}{2} m_{\text {IDSHO }}\left(\frac{\Delta E}{\eta}\right)^{2} y^{2}+V_{o}
$$

Taking Eq. (11) into account Eq. (9)), it will become $\frac{d^{2} u}{d y^{2}}+q\left[E-V_{o}-\frac{q}{4} m_{I D S H O}\left(\frac{\Delta E}{\eta}\right)^{2} y^{2}\right] m_{I D S H O} u=0$

Eqs. (7) and (12) must coincide and Eqs. (9) and (12) must also coincide. Then their solutions can be obtained respectively as

$$
m(z)=m_{B C} \operatorname{Cosh}^{2}\left(\frac{\Delta E}{\eta} \sqrt{\frac{q}{\phi} z}\right)
$$

and

$$
V(z)=\phi_{B C} \operatorname{Sinh}^{2}\left(\frac{\Delta E}{\eta} \sqrt{\frac{q}{\phi} z}\right)
$$

Now taking into account Eqs. (4) and (3), the grading function becomes: 


$$
x(z)=\frac{m_{B C}}{\Delta m} \operatorname{Sinh}^{2}\left(\frac{\Delta E}{\eta} \sqrt{\frac{q}{\phi} z}\right)
$$

Eqs. (13) - (15) are the desired electron effective mass potential experienced by the electron and the grading function respectively to achieve a physically realizable equispaced energy level QW ternary semiconductor. The wave function corresponding to the eienstates is given by:

$$
\psi_{1}(z)=\left(\frac{1}{i l 2}\right)^{1 / 2}[q \Delta E m]^{1 / 4} U_{I}(z)
$$

where the eigenfunctions $\mathrm{U}(\mathrm{z})$ are the well-known Hermite functions.

$U_{1}(z)=\left(\frac{1}{i l 2}\right)^{1 / 2}[q \Delta E m(z)]^{1 / 4} H_{I}(z) e^{-\frac{1}{2}(z)}$

For $\mathrm{i}=0,1,2$;

$$
H_{o}(z)=1, H_{1}(z)=2 z \text { and } H_{1}(z)=4 z^{2}-2
$$

Thus the corresponding eigenfunctions are

$$
\begin{aligned}
& \psi_{o}(z)=\left[q \Delta E m_{B C}\right]^{1 / 4} \operatorname{Cosh}^{1 / 2}\left(\frac{\Delta E}{2} \sqrt{\frac{q}{\phi}} z\right) e^{-\frac{1}{2}(z)}(19) \\
& \psi_{1}(z)=2\left[\frac{1}{4} q \Delta E m_{B C}\right]^{1 / 4} \operatorname{Cosh}^{1 / 2}\left(\frac{\Delta E}{2} \sqrt{\frac{q}{\phi}} z\right) e^{-\frac{1}{2}(z)^{2}}
\end{aligned}
$$

$$
\psi_{2}(z)=\frac{2}{2}\left[\frac{1}{4} q \Delta E m_{B C}\right]^{1 / 4} \operatorname{Cosh}^{1 / 2}\left(\frac{\Delta E}{2} \sqrt{\frac{q}{\phi}} z\right)\left(2 z^{2}-i\right) e^{-\frac{1}{2}(z)^{2}}
$$

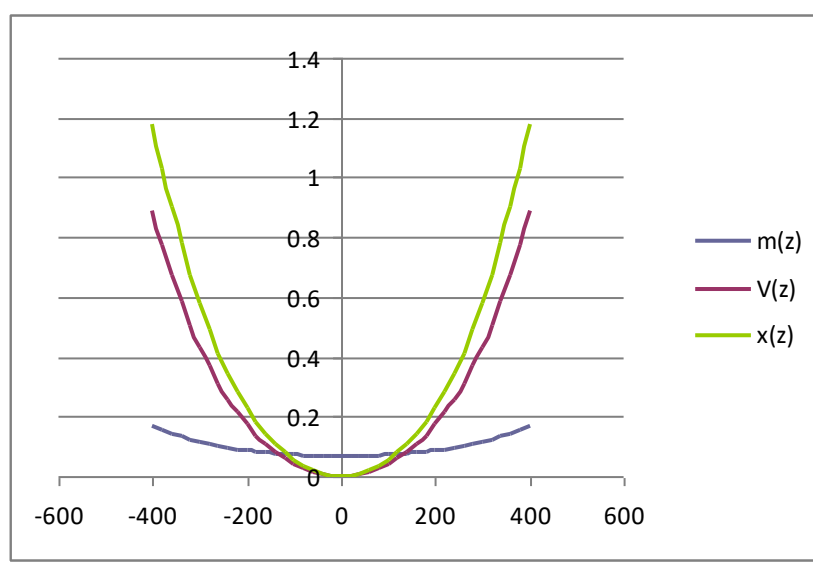

Fig la: The Effective mass $m(z)$, the potential $v(z)$ and the mole fraction $x(z)$ for $A l_{x} G a_{l_{-x}} A s$

\section{RESULTS AND DISCUSSION}

The calculation of the electron effective mass grading function potential and wave functions are performed by a method that has been previously described [15] the election effective mass the potential and the wave functions (at i =0 1 and 2) are obtained from Eq.S (213) (219) (220) and (221) respectively using a common value in the literature for $\Delta \mathrm{E}=30 \mathrm{meV}$ [22] the values of the material effective masses and minimum band gaps are obtain from David (1991) [27] and band off-set (the last column of Table 1) from $[14,15]$. Graphs of the electron effective mass and potential dependence on the chosen direction of confinement for nine ternary semiconductors QWs. All the graphs of Fig 1a to Fig 9a are parabolic and have equispaced states both for the effective mass and potential with that of the former having a wilder spread.

The trend here is that the grading function which linearly depends on the conductor band edge hence the potential has a linear relation with the electron effective mass [18]. Since band edge dependence on material composition is indispensible in band gap engineering [23], the equispaced states electron effective mass will be very useful in obtaining equispaced energy levels in QWs.

The calculated dependence of the normalized wave functions $\mathrm{U}(\mathrm{z})$ of the first three bound states (Figs. 1 (b) to 9(b)) on the direction of confinement provides as a check on our formulation. For it is a common knowledge that irrespective of the variation of the energy levels, the sinusoidal path of the wavefunction for each of the level should not be affected. Therefore the achievement of the expected path for the wave function from our formulation implies that our coordinate transformation was successful.

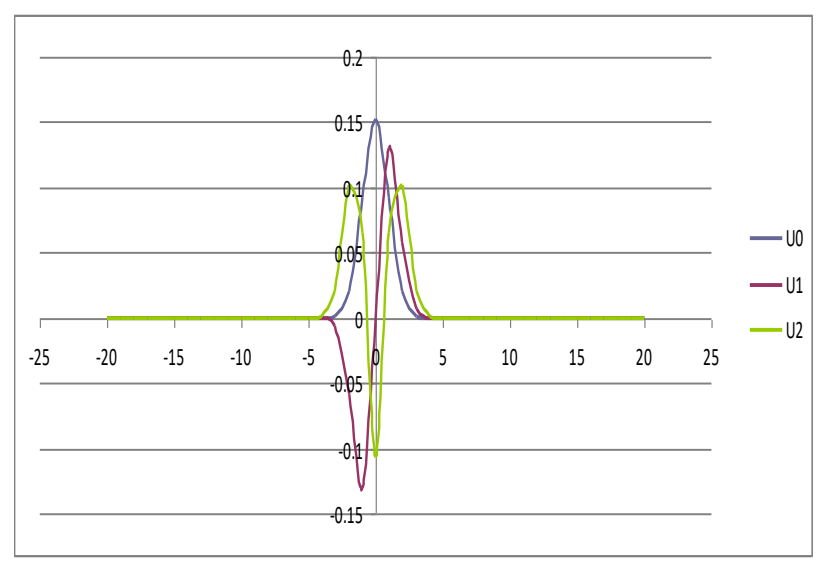

Fig. 1b: The normalized wave functions $U i(z)$ of the first three bound state with $\in=0$ for $A l_{x} G a_{1-x} A s$ 


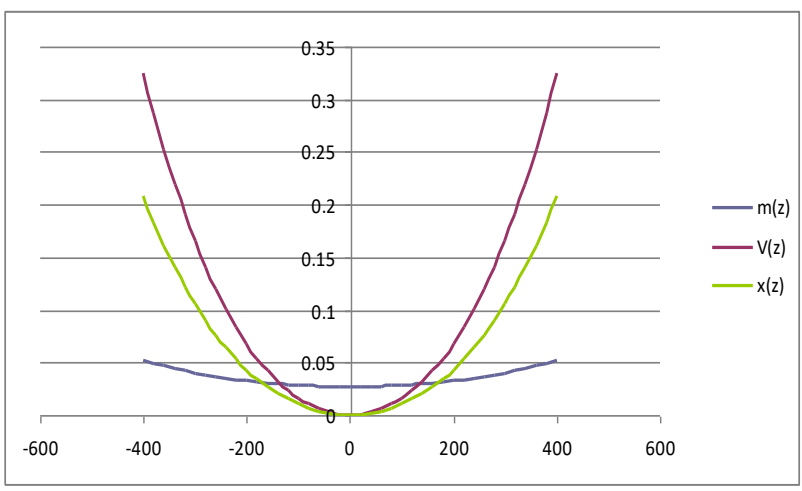

Fig 2a: The Effective mass $m(z)$, the potential $v(z)$ and the mole fraction $x(z)$ for $A l_{x} I_{1-x} A s$

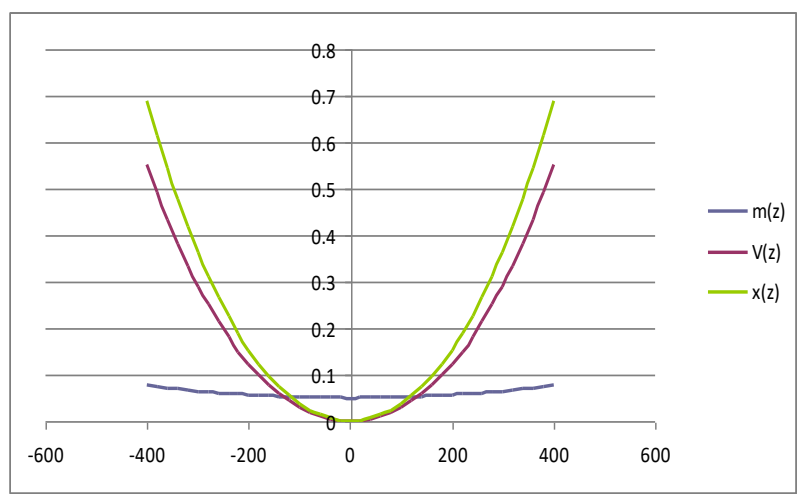

Fig 3a: The Effective mass $m(z)$, the potential $v(z)$ and the mole fraction $x(z)$ for $A l_{x} G a_{1-x} S b$

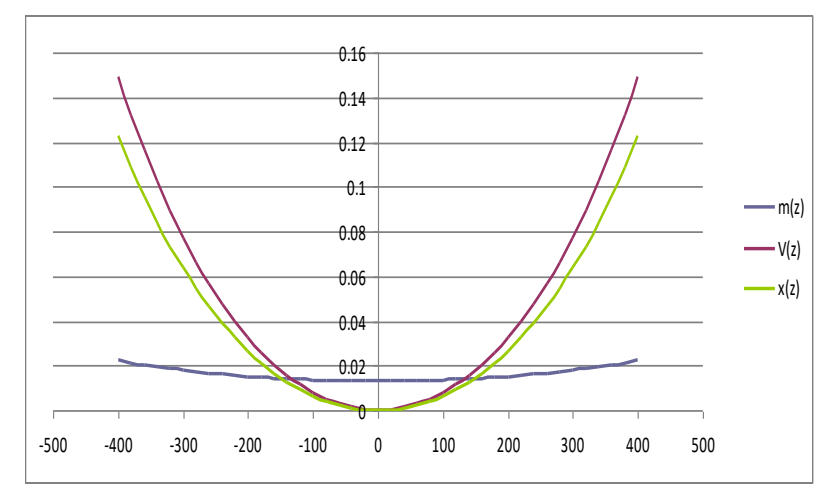

Fig4a: The Effective mass $m(z)$, the potential $v(z)$ and the mole fraction $x(z)$ for $A l_{x} I n_{1-x} S b$

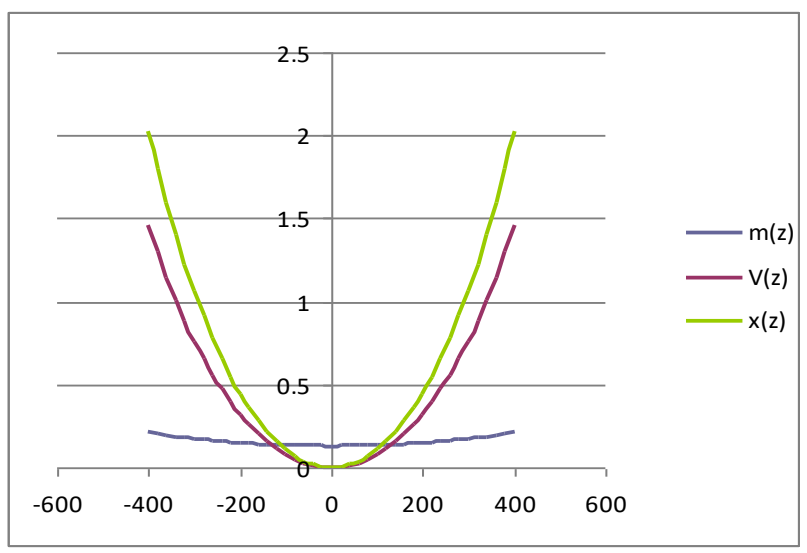

Fig 5a: The Effective mass $m(z)$, the potential $v(z)$ and the mole fraction $x(z)$ for $C d_{x} Z n_{1-x} S e$

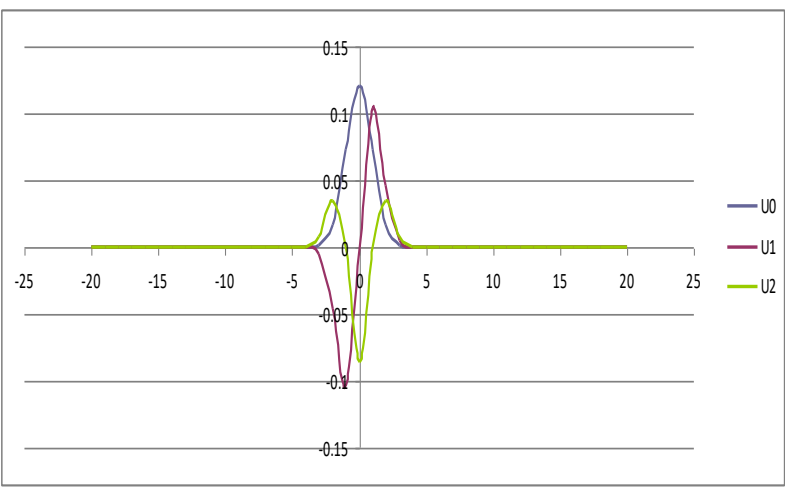

Fig. 2b: The normalized wave functions $U i(z)$ of the first three bound state with $\in=0$ for $A l_{x} \operatorname{In}_{1-x} A$ s

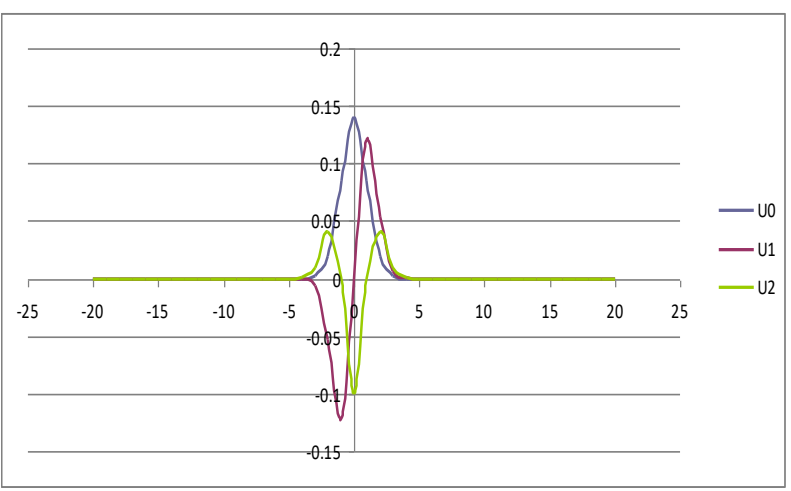

Fig. 3b: The normalized wave functions $U i(z)$ of the first three bound state with $\in=0$ for $A l_{x} G a_{1-x} S b$

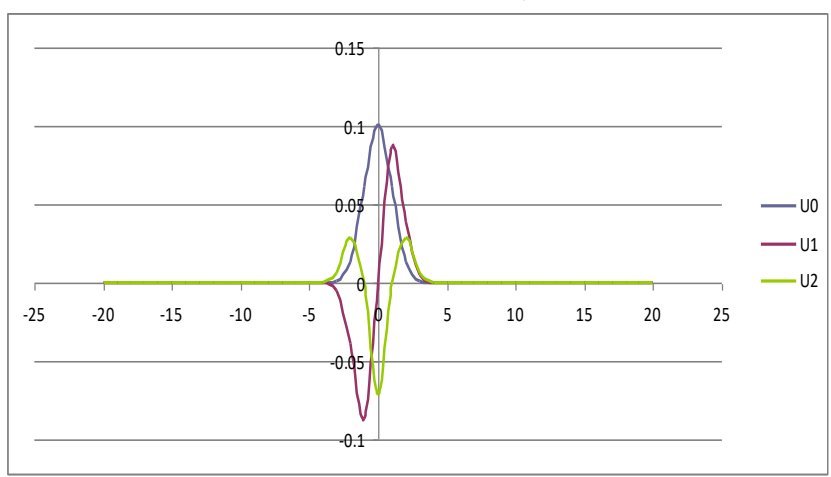

Fig.4b: The normalized wave functions $U i(z)$ of the first three bound state with $\in=0$ for $A l_{x} I_{1-x} S b$

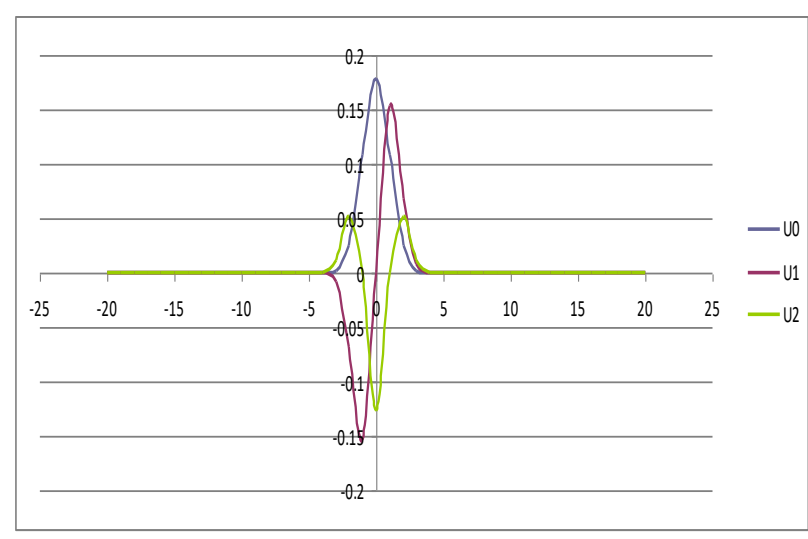

Fig. 5b: The normalized wave functions $U i(z)$ of the first three bound state with $\in=0$ for $C d_{x} Z n_{1-x} S e$ 


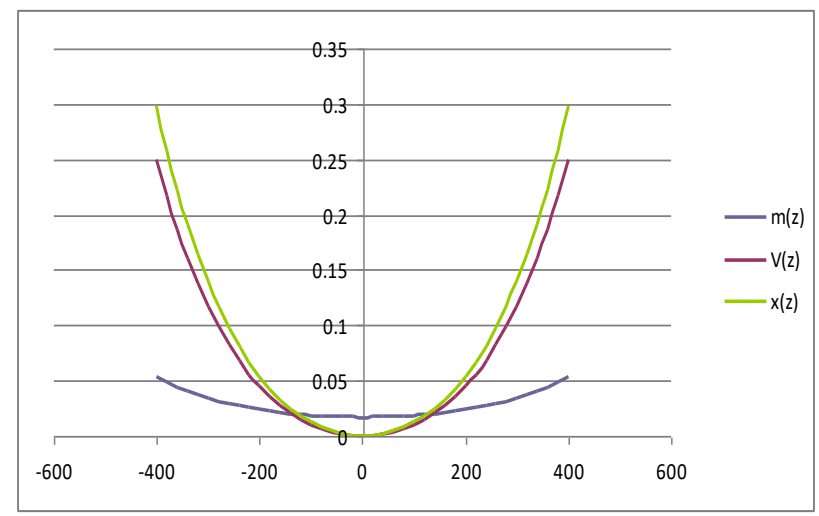

Fig 6a: The Effective mass $m(z)$, the potential $v(z)$ and the mole fraction $x(z)$ for $\mathrm{Cd}_{x} \mathrm{Hg}_{1-x} \mathrm{Te}$

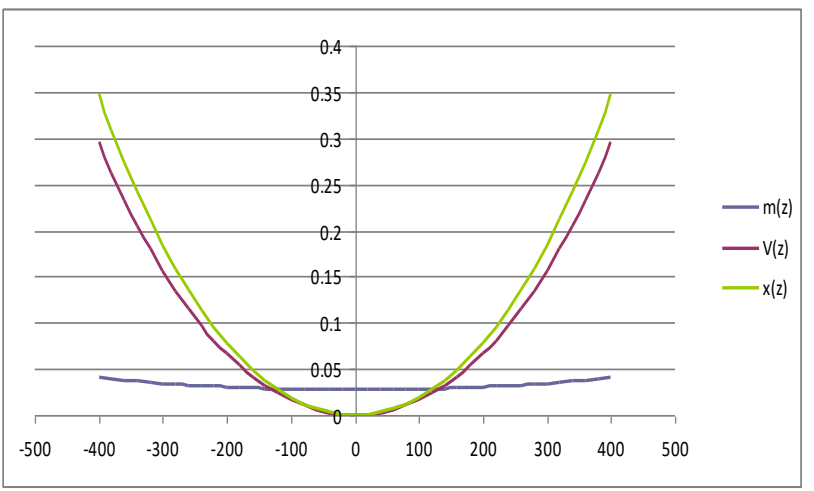

Fig7a: The Effective mass $m(z)$, the potential $v(z)$ and the mole fraction $x(z)$ for $G a_{x} I n_{1-x} A s$

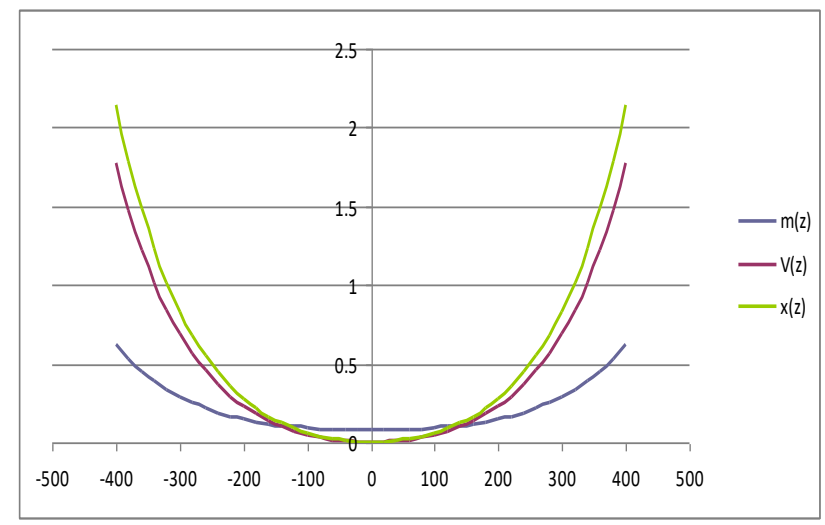

Fig 8a: The Effective mass $m(z)$, the potential $v(z)$ and the mole fraction $x(z)$ for $G a_{x} I n_{1-x} P$

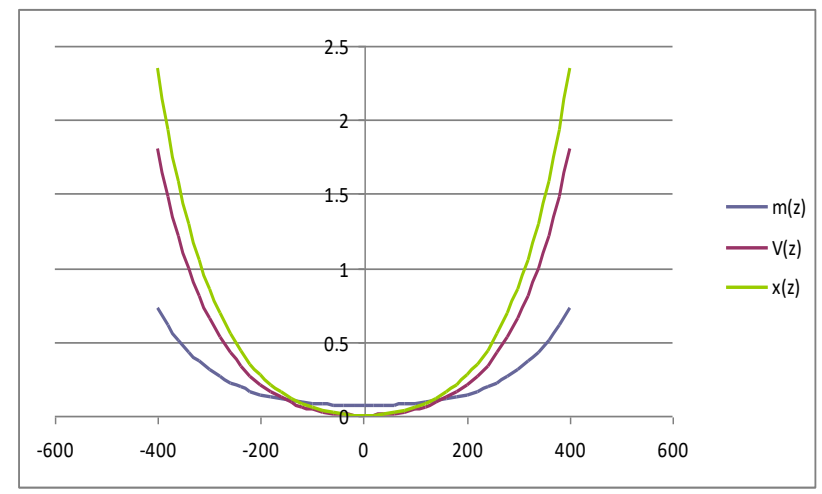

Fig. 9a: The Effective mass $m(z)$, the potential $v(z)$ and the mole fraction $x(z)$ for $G a_{x} P_{1-x} A s$

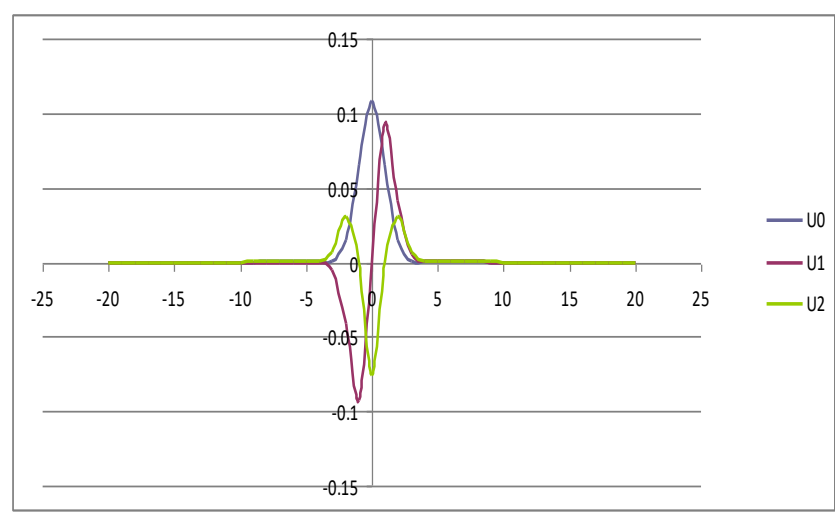

Fig. 6b: The normalized wave functions $U i(z)$ of the first three bound state with $\in=0$ for $\mathrm{Cd}_{x} \mathrm{Hg}_{1-x} \mathrm{Te}$

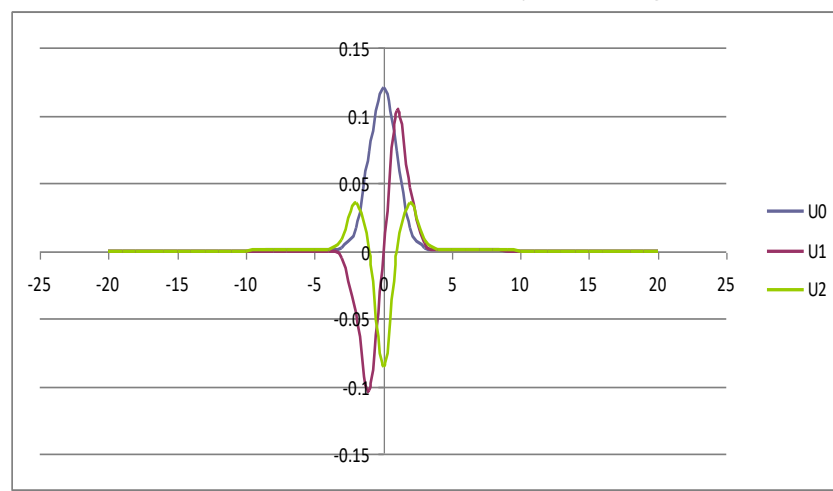

Fig.7b: The normalized wave functions $U i(z)$ of the first three bound state with $\in=0 G a_{x} \operatorname{In}_{l_{-x}} A$ s

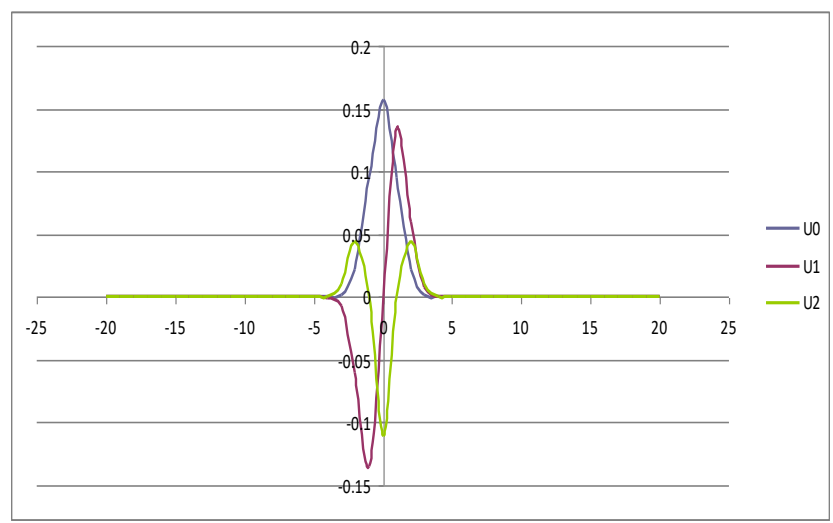

Fig.8b: The normalized wave functions $U_{i}(z)$ of the first three bound state with $\in=0 \quad G a_{x} \operatorname{In}_{1-x} P$

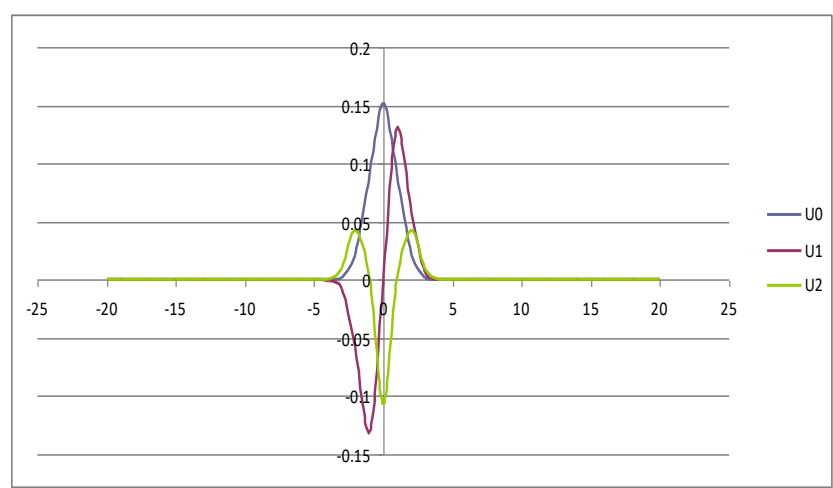

Fig. 9b: The normalized wave functions $U i(z)$ of the first three bound state with $\in=0 G a_{x} P_{1-x} A$ s 
Table 1: Some Semiconducting Properties of Selected Ternary Alloy [27]

\begin{tabular}{|c|c|c|c|c|}
\hline & \multirow{2}{*}{$\begin{array}{l}\text { Semiconductor alloy system (Ternary } \\
\text { alloy); } A_{x} B_{1-x} C\end{array}$} & \multicolumn{2}{|c|}{$\begin{array}{c}\text { Electron Effective Mass }\left(\mathrm{M}_{0}\right) \text { and Minimum } \\
\text { band gap }(\mathrm{eV})\end{array}$} & \multirow{2}{*}{$\begin{array}{l}\text { Band off set } \\
\quad(\mathrm{meV})\end{array}$} \\
\hline & & $M_{B C}$ & $M_{A C}$ & \\
\hline & & Ga As & Al As & \\
\hline \multirow[t]{3}{*}{1} & GaAs/AlAs $\left(\mathrm{Al}_{x} \mathrm{Ga}_{1-\mathrm{x}} \mathrm{As}\right)$ & $0.067 \mathrm{mo}_{0}$ & $0.15 \mathrm{mo}_{0}$ & \\
\hline & & $1.35 \mathrm{eV}$ & $2.2 \mathrm{eV}$ & rsumev \\
\hline & & $\mathrm{Cd} \mathrm{Se}$ & ZnSe & \\
\hline \multirow[t]{3}{*}{2} & $\mathrm{ZnSe} / \mathrm{CdSe}\left(\mathrm{Cd}_{x} \mathrm{Zn}_{1-\mathrm{xSe}}\right)$ & $0.13 \mathrm{mo}_{0}$ & $0.17 \mathrm{mo}_{0}$ & $730 \mathrm{meV}$ \\
\hline & & $1.74 \mathrm{eV}$ & $2.58 \mathrm{eV}$ & I $\angle 0$ mev \\
\hline & & $\mathrm{Hg} \mathrm{Te}$ & $\mathrm{Cd} \mathrm{Te}$ & \\
\hline \multirow[t]{3}{*}{3} & $\mathrm{HgTe} / \mathrm{CdTe}\left(\mathrm{Cd}_{x} \mathrm{Hg}_{1-\mathrm{x}} \mathrm{Te}\right)$ & $0.017 \mathrm{mo}_{0}$ & $0.14 \mathrm{mo}_{0}$ & $840 \mathrm{meV}$ \\
\hline & & $0.15 \mathrm{eV}$ & $1.44 \mathrm{eV}$ & otumev \\
\hline & & In As & Al As & \\
\hline \multirow[t]{3}{*}{4} & In As/AlAs (Al $\left.x_{x} I_{1-x} A s\right)$ & $0.027 \mathrm{~m}_{0}$ & $0.15 \mathrm{~m}_{0}$ & \\
\hline & & $0.36 \mathrm{eV}$ & $2.2 \mathrm{eV}$ & $150 / \pi$ III \\
\hline & & Ga Sb & $\mathrm{Al} \mathrm{Sb}$ & \\
\hline \multirow[t]{3}{*}{5} & $\mathrm{GaSb} / \mathrm{Al} \mathrm{Sb}\left(\mathrm{Al}_{x} \mathrm{Ga}_{1-\mathrm{x}} \mathrm{Sb}\right)$ & $0.050 \mathrm{mo}_{0}$ & $0.09 \mathrm{mo}_{0}$ & $800 \mathrm{meV}$ \\
\hline & & $0.67 \mathrm{eV}$ & $1.6 \mathrm{eV}$ & ovoninev \\
\hline & & In Sb & $\mathrm{Al} \mathrm{Sb}$ & \\
\hline \multirow[t]{3}{*}{6} & In $\mathrm{Sb} / \mathrm{Al} \mathrm{Sb}\left(\mathrm{Al}_{\mathrm{x}} \mathrm{In}_{1-\mathrm{x}} \mathrm{Sb}\right)$ & $0.013 \mathrm{mo}_{0}$ & $0.09 \mathrm{mo}_{0}$ & 1220 \\
\hline & & $0.165 \mathrm{eV}$ & $1.6 \mathrm{eV}$ & \\
\hline & & In $\mathrm{P}$ & Ga P & \\
\hline \multirow[t]{3}{*}{7} & In $\mathrm{P} / \mathrm{Ga} \mathrm{P}\left(\mathrm{Gax}_{\mathrm{x}} \mathrm{In}_{1-\mathrm{x}} \mathrm{P}\right)$ & $0.077 \mathrm{~m}_{0}$ & $0.35 \mathrm{mo}_{0}$ & 825 \\
\hline & & $1.27 \mathrm{eV}$ & $2.24 \mathrm{eV}$ & $0<0$ \\
\hline & & In As & Ga As & \\
\hline \multirow[t]{3}{*}{8} & In As/Ga As (Gax In $\left.\operatorname{In}_{1-x} \mathrm{Sb}\right)$ & $0.027 \mathrm{mo}_{0}$ & $0.067 \mathrm{~m}_{0}$ & 850 \\
\hline & & $0.36 \mathrm{eV}$ & $1.35 \mathrm{eV}$ & 850 \\
\hline & & Ga As & Ga P & \\
\hline \multirow[t]{2}{*}{9} & Ga $P_{x} A s_{1-x}$ & $0.067 \mathrm{~m}_{0}$ & 0.35 & 770 \\
\hline & & $1.35 \mathrm{eV}$ & $2.24 \mathrm{eV}$ & (1) \\
\hline
\end{tabular}

\section{CONCLUSION}

QW and nanostructures generally are broadly tailorable to various the design [24]. Which find applications in optoelectronics such as infrared photodetectors, infrared imagine, laser photodetectors and diode lasers [25]. All these devices operates optimally with coherent emission. One approach to achieve coherent emission is to design these devices with equispaced energy level QW. In this study, we have demonstrated a generalized method to obtain equispaced states of the electron effective mass and potential as signatures of equipment energy levels for QWs in semiconductor ternary alloys. As stated above, the calculated electron effective masses can be used as inputs for further single band calculations to obtain the electronic structure when the absolute size of the structures is known $([23,25]$. The generalized formulation here can be extended to other semiconductors nanostructures such as theoretical design of equispaced energy level quantum wires which are two-dimensional confinement [26] and quantum dots which are three-dimensional confinement [13]. It is expected that the equispaced energy level will reduce the effect of decoherence in these semiconductors nanostructures especially in their applications that require coherent emission and absorption. Further, electronic devices such as the resonant tunneling diode are also affected by the position-dependence of carrier effective mass and thus the results are applicable to both optoelectronic and electronic quantum devices with real materials but also with ultracold atoms in optical lattice for the effective mass and potential with that of the former having a wider spread. 


\section{REFERENCES}

[1] Dingle R, Wiegmann G. and Henry C.H . Quantum state of confined carriers in very thin $\mathrm{Al}_{\mathrm{x}} \mathrm{Ga}_{\mathrm{x}}$ AsGaAs-Al $\mathrm{Ga}_{x}$ As heterostructures. Phys. Rev. Lett. 33, 1974, pp 827-830.

[2] Ejere A. Equispaced, level quantum well and semiconductor alloy nanostructures. Lambert Academic. Publishing (LAP), Germany. Online@www.lap-publishin.com ,2011.

[3] Harrison P. Quantum wells, wires and dots Theoretical and computational physics of semiconductor nanostructures Wiley interscience, John Wiley and sons Ltd,2005.

[4] Taylor J.M Engel H.A.. Dur W.. Yacoby A... Marcus C.M > Zoller $\mathrm{P}$ and Lukin M.D. Faultotolerant architecture for quantum computation using electrical controlled semiconductor spins, Nature Phys. 1, 2005, pp177-184.

[5] Kumar K,N.P.. Kirnaji B.S and Bagewadi C.S. Decohering environment and coupled quantum state and internal resonance in coupled spin system and the conflict between quantum gate operation and decoupling a Cormorant-Barnacle model, Adv. In Phys. Theories and Application 6, 2002 pp 24-31.

[6] Schlosshaurer $M$. Decohenrence, the measurement problem and interpretation of quantum machenes, Rev. Mod Phys. 76, 2004, pp 1267-1274

[7] Golubev D.S. and Zaikin A.D. Quantum decoherence of interacting electrons in arrays of quantumdots and diffusive conductors, anXiv 08073227, 2008.

[8] Kucharska A.C. and Robbing D.J. (1990) Lifetime broadening in GaAs-AlGaAS quantum well IEEE Journal of Quantum Electronics 26, 1990, pp 443451.

[9] Shockey W US patent 2, 569, 347, 1951

[10] Kromer H. b Proceeding of IRE 45,1957 pp 15351542. [11] Coish W.A and Daniel Loss Singlettripled decoherence due to nuclear spins in a quantum dot, Phys. Rev Lett. 72, 2005 pp 125337125344

[12] Fisher J. and Loss D. Dealing with Decoherence, Science 324, 2009 pp 1277-1284.

[13] Akpojoptor G.E. and Akpojotor F.A. A highly simplified correlated variational study of the singlet-triplet transition in quantum dots embedded in kagome lattice, AIP on. Proc.11, 2009, pp 40.54.

[14] Milanovic V. and Ikonic Z., Equispaced-level Hamiltonian with veriable effective mass following the potential, Phys. Rev. B. 54, 1996, pp998-1006.

[15] Milanovic V., Ikonic Z. and Indjin D. Optimization of resonant intersubband nonlinear optical susceptibility in semiconductor quantum wells: the coordinator transform approach Phys. Rev. B.53, 1996, pp10887-10895.

[16] Ejere I.I.A. and Idiodi J.O.A Equinspaced level conduction band design in the $\mathrm{CdZnSe} / \mathrm{CdSe}$ quantum well: JPS 6(3), 2011 pp 500-505.

[17] Ejere A. and Akpojotor G.E. A theoretical design of quantum wells with equispaced energy level in the condition band of $\mathrm{Al}_{\mathrm{x}} \mathrm{Ga}_{1-\mathrm{x}} \mathrm{Sb}$, Afr. J. of Phys. 4, 2011 pp 62-82.

[18] Radovanovic J. Millanovie wells. Phys Letts A269, 179.

[19] Einevoll G.T., Hemmer P.C and Thomsen J. Operator ordering in effective-mass theory for heterostructures. 1. Comparison with exact results for superlattices, Phys. Rev. B. 42, 1990, pp 34853493.

[20] Eugene B. Mathematical Physics. Addison-Wesley Publishing Company, 1970.

[21] Abramowitz M. and Stegun I.A. Handbook of mathematical function Dover publisher, Inc, New York, 1972.

[22] Qi Y. and Qi Y Optical interface phonon modes ingrades quantum well (GQS)n structure perturbation theory J. Phys. Condens. Matter 11. 1999, pp 8855-8864

[23] Pryor. C.E and Pistol M.E. Band-edge diagram for a strained III-V semiconduct quantum wells, wires and dots. arXiv:cond-mat/0501090, 2005.

[24] Numikko A.V. and Gunshor R.L. (1994) BlueGreen emitter in Wide-gap II-IV quantumconfined structure, IEEE journal of Quantum Electronics 30, 1994 pp 619-628.

[25] Imam N., Brasil M.J.S.P.. Brum J.A., Poole P., Charbonnean S., Tamargo M.C. Excition dynamics in a single quantum well with self-assembled Island Phys. Rev. B. 53, 1996, pp. 16524-16533.

[26] Yang Y. Fedorov G. Barbara P., Shafranjuk S.E., Cooper B.K., lewis R.M and Lobb C.J. (2003) Coherent nonlocal transport in quantum wires with strongly coupled electrodes, Phys. Rev. B87, 2003, pp. 045403-045412.

[27] David R.L. Semi-conducting properties of selected materials, CRC handbook of Chemistry and Physics (71 ${ }^{\text {st }}$ Ed). CRC Press, New York, pp12-62, 1991. 